\title{
Case Study: The Benefits of Integrating 3D Printing into Prosthetics by Comparing a Traditional Manufactured Prosthesis to a 3D Manufactured Transradial Prosthesis
}

\author{
Courtney Mortier ${ }^{1}$ and Kimberly Tetmeyer ${ }^{1 \#}$ \\ ${ }^{1}$ Lapeer High School, Lapeer, MI, USA \\ \#Advisor
}

$\underline{\text { ABSTRACT }}$

This research study will be looking at the benefits and time-frame benefits of 3D printing. The purpose of this is to prove how the data collected agrees with past research studies on how the integration of 3D printing technology into the field of orthotics and prosthetics is considered beneficial. Participant one was given two sets of 10 questions to find a statistical significance between a traditional transradial prosthesis and a 3D manufactured transradial prosthesis. By doing this I then compared participant one's answers to a similar survey given to participants two and three, then drew the connection of how it is much more beneficial while also accounting for confounding variables. Consequently, this study concluded that the majority of answers benefited the integration of 3D printing. It proved this by having the participant explain their answers and also compare a traditional prosthesis to a 3D printed prosthesis specifically explaining the time-frame difference and the benefits of having the digitally manufactured prosthesis. This indicated how my research did support the past research done on this specific topic on how it is beneficial that 3D manufacturing should be integrated into prosthetics and orthotics. This study is solely correlational and analyzed data, meaning, it does not actually prove anything but was simply done to show the connection of the topic. With the results of this research, it will add more support to the current body of research.

\section{Introduction: J.E. Hanger Story}

The design of functional prosthetic devices can be traced back to the Ancient Roman and Egyptian empires, since then our world has continuously worked to develop many more functional prostheses throughout the many decades that have passed. Going back to John Hanger, at this time he was an 18-year-old engineering student at Washington College (now Washington and Lee University) [4]. He decided to join his siblings in the early days of the Civil War, and within two days of being enlisted, a cannonball took his leg in the Battle of Philippi. He was then documented as the first amputee of the Civil War after surviving an excruciating amputation that in this time period was necessary to save his life.

Becoming a patient in the field of orthotics and prosthetics puts anyone on quite an embarking journey, this journey includes permanent changing of an individual's perspective, body image, and spirit. An unnumbered number of circumstances can take a toll on an individual's limb including: congenital deformities, diseases, diabetes, countless illnesses, and various traumatic injuries. Hanger started to invent and design numerous legs (figure 1) which, in modern times, have proven to be revolutionary in the field of prosthetics and orthotics [4]. When he reflected on this time in his life, he was quoted by speaking. "No one can know what such a loss means unless he has suffered a similar catastrophe. In the twinkling of an eye, life's fondest hopes seemed dead, What could the world hold for a maimed, crippled man" [2]. 
From this point he developed the "Hanger Limb". He then quoted "Today I am thankful for what seemed then to me nothing but a blunder of fate, but which was to prove instead a great opportunity" [6]. Throughout this paper, the goal was to prove that $3 \mathrm{D}$ printing is more beneficial than a traditionally built prosthesis by comparing the two methods through a case study. In the paper it will discuss three patients, P2 and P3 being from another source [1], and my primary patient P1. For reference, P1 represents my primary data in this research. The goal for P1 was to use both a traditionally built arm and the digital arm I designed. For the results P1 is to compare both arms and describe why the preference is greater for one than the other, and for a broader perspective, my secondary data of P2 and P3 are of contrasting age to show how it is not only young adults who prefer digitally printed prostheses. By comparing the results, I get from this research, I will hopefully be able to clearly see the benefits of digital manufacturing for prosthetics.

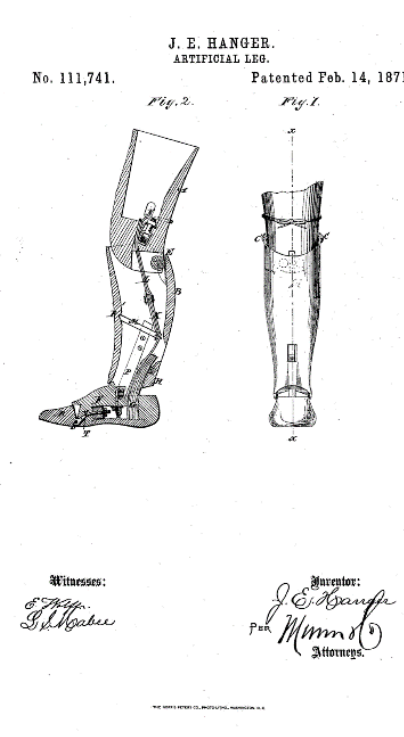

A.

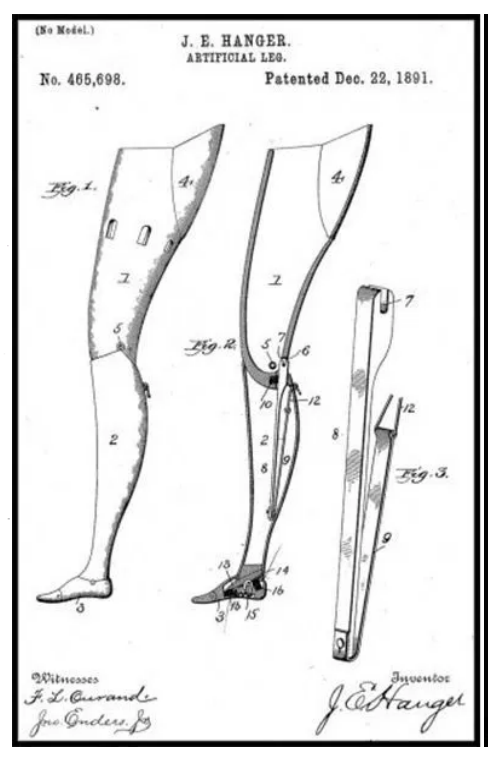

B.

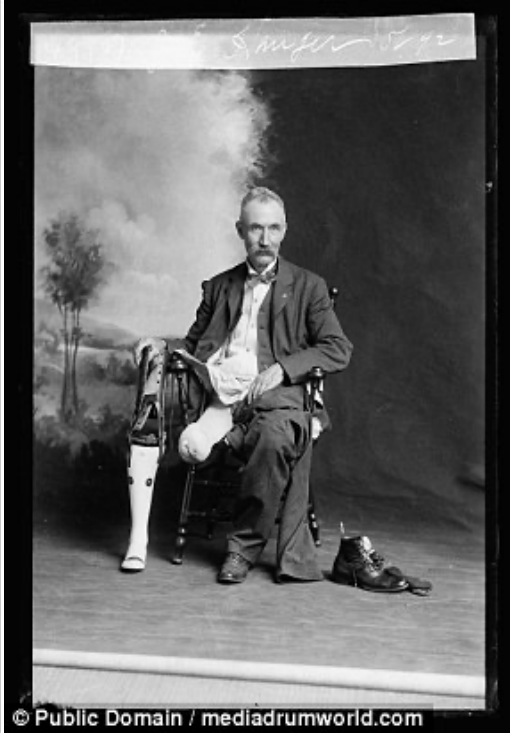

C.

Figure 1: Early designs of Hanger prosthetic legs (A and C). Image of J.E. Hanger after his amputation (B).

\subsection{Literature Review}

Various media reports and research papers and researchers have dedicated their time in attempting to explain their view of misconceptions with integrating 3D printing to manufacture limbs in the field of orthotics and prosthetics [1]. Although this point of view is far from reality within the medical community, the opinion still gets attention. Very few elements of digital manufacturing were introduced to clinics over thirty years ago according to [1]. This paper will focus mainly on P1 but will also add in commentary on P2 and P3 which are from [1]. This research paper will aim to:

- Explain the process and benefits of making a 3D printed limbs on an Ender 3 V2 printer [6]

- Present patient feedback on both the original upper-limb prosthesis and the digital version

In my research, I will attempt to show the comparison results of the benefits of a 3D manufactured transradial prosthesis with a traditionally built transradial prosthesis. More recently digital manufacturing has become admired in the field of prosthetics. Minimum prior research has been done on this topic, as well as other researchers having difficulty achieving the positive goal of the topic. In a study done by Jen Olsen et al [1], she reviewed digital prosthesis outcomes 
on her patients. Olsen's outcomes did turn out positive to the topic and correlated with my body of research by her discussion of why her patients preferred the digital prosthesis. Referenced below is a table manifesting the three patients with relative information about each individual.

Table 1: Referenced from [1] Shows participant gender (self-identified; age-range; cause (A: Amputation, C: Congenital, IL: illness, IJ: injury); Prosthesis type (P: passive, BP: body powered, M: myoelectric); Frequency of use (Frequent, daily, occasional, none); and notes (either what their complaint is (PLS: phantom limb sensations, P: pain, OG: outgrew; or which plastic is used (PLA or ABS or Traditional arm TA). P1 is primary data, P2-P3 is secondary data [1].

\begin{tabular}{|c|c|c|c|c|c|l|}
\hline Participant & Gender & Age Range & Cause & $\begin{array}{c}\text { Prosthesis } \\
\text { Type }\end{array}$ & $\begin{array}{c}\text { Prosthesis } \\
\text { Use }\end{array}$ & \multicolumn{1}{|c|}{ Notes } \\
\hline P1 & Male & $14-17$ & C & BP & Occasional & $\begin{array}{l}\text { OG, P, Pri- } \\
\text { mary data, } \\
\text { TA }\end{array}$ \\
\hline P2 & Male & $50-60$ & A & P/BP & Occasional & $\begin{array}{l}\text { PLS, P, sec- } \\
\text { ondary data } \\
\text { [1] }\end{array}$ \\
\hline P3 & Female & $40-50$ & A & P/BP & Occasional & $\begin{array}{l}\text { P, PLS } \\
\text { (mild), sec- } \\
\text { ondary data } \\
\text { [1] }\end{array}$ \\
\hline
\end{tabular}

Small modifications can make all the difference when trying on the final fit of the socket. During the traditional process of upper-limb socket making, it is calamitous. To capture the true dimensions of the limb is very restraining throughout the entire casting process, and in the case of having no backup plan for rectifying mistakes, this whole casting process must be repeated until done correctly, which requires the patient to sit through the process again in the clinic taking more of their time. Not to mention making an error during the traditional process is an inconvenience for anyone involved, coming from a clinical standpoint, replacing the mistaken socket entails more labor and is very timeconsuming to fix errors in the original casting with a limited financial return for the added hours of labor and having to go through more material [3]. Going into digital manufacturing, if this was an issue with a 3D printed limb it could be fixed within hours or minutes. An example of this could be, if the finger had too much filament where it needed to bend, a single finger to print could easily take 5-20 minutes depending on printing speed, whereas in a traditional build it is more than likely the patient would have to come back on a different day, once the issue is corrected.

\section{Methods}

The methods sections of this case study paper will identify ethics as well as explain the experiment with the three participants. It will mainly focus on P1, considering it is primary data, again, $\mathrm{P} 2$ and $\mathrm{P} 3$ are being used for comparison and are pulled from [1]. Images will be presented to allow for a better understanding of a transradial prosthesis and where exactly it pinpoints on the body (figure 2). Further down in the section (2.3) there will be a brief overview on why traditional builds are inconvenient compared to a digital method, as well as explaining the similar steps of both but still identifying why 3D printing should be integrated into the field of O\&P. After this section will be section 2.4, which will go over the printing process of the limb and the interview process and show what was given to P1 and was similarly given to $\mathrm{P} 2$ and $\mathrm{P} 3$. 


\subsection{Ethics}

Local AP Research: Capstone teacher approved of this study and CollegeBoard

\subsection{Participants}

Three participants who had a transradial limb absence were considered for this experiment, all of different or similar causes. Two of the Three participants reported the use of a 3D printer, one reported modification with a 3D printer. Additional data of further participants can be found in Table 1.
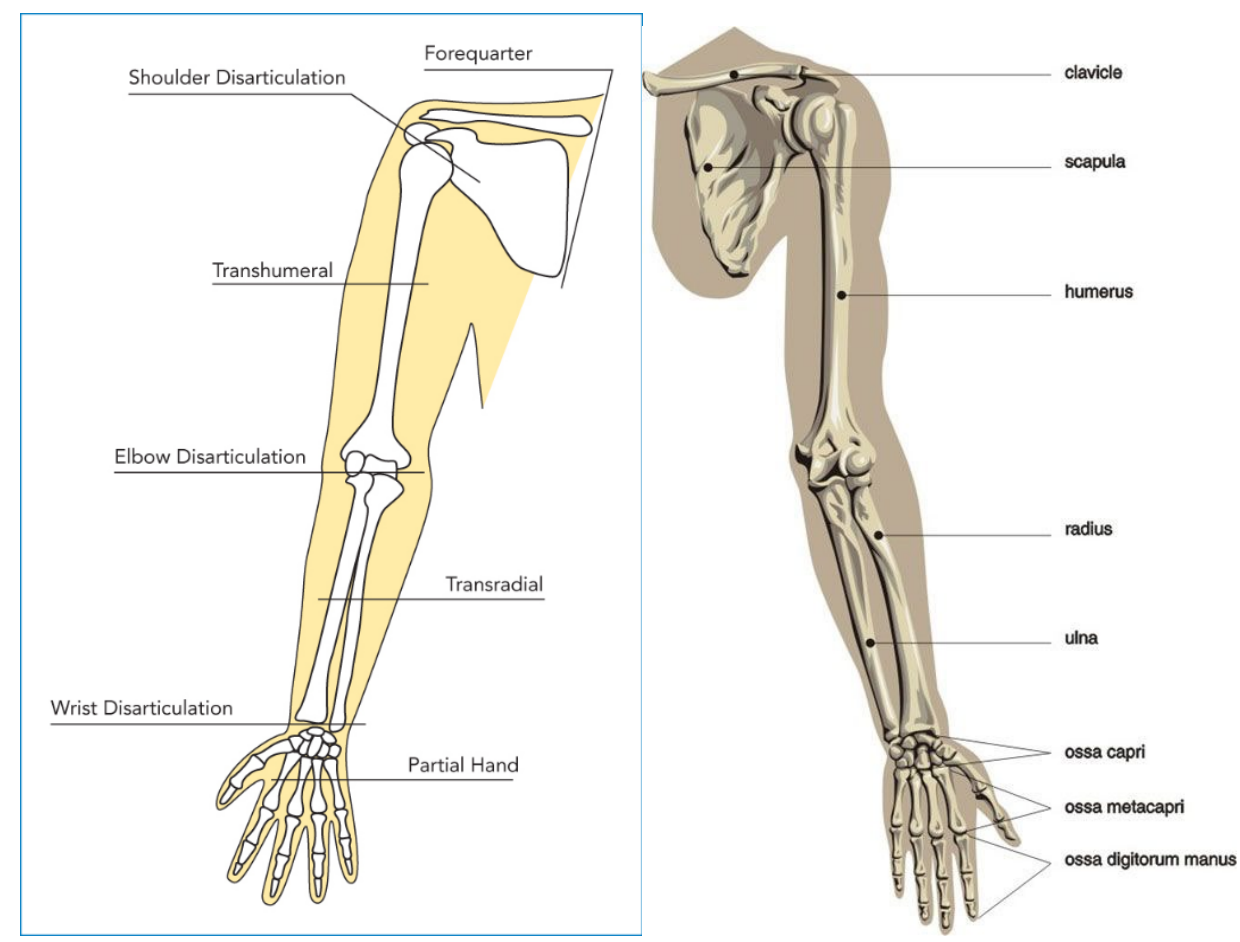

Figure 2: showing the anatomy of the arm and socket terminology for clarity.

\subsection{Why the traditional build of upper-limb sockets is considered inconvenient: Method}

I will be using a mixed quantitative and qualitative correlational analysis method to look at the benefits of digital manufacturing, with seeing the association between traditional transradial manufacturing to a digitally manufactured transradial prosthesis. Furthermore, I will analyze then apply my results using this method to compare the two manufacturing processes. My research is a mixed quantitative and qualitative correlational analysis because I conducted an experiment, then analyzed my findings and results. For better explanation, I used my primary data (P1) by asking them 10 questions (as seen in 2.4). A brief explanation of those includes asking the patient which arm was more comfortable and which was shown to be more beneficial. I created this sequence of questions, then instructed P1 to estimate the time-frame difference between the delivery of both arms. I then analyzed the results of the responses in an attempt to find the correlation between the various categories of questions. These steps were the general layout of how my research was conducted. 
My participants are of three individuals who had either an amputation or a congenital deformity. Their age ranges from 14 through 60 years old (table 1). Participant one was referred to as P1, patient P2 and P3 we're pulled to be compared to my primary data P1 from research done by Olsen [1]. I chose to look at different age groups because as mentioned in my literature review, I wanted there to be a broader perspective on why digital manufacturing is more beneficial not only in young adolescents but in adults as well. Due to all three patients having prior experience to having a traditional transradial prosthesis, and now having one that was digitally manufactured, this is what led me to the gap I am focusing on. The Research I am doing may limit my results because I can only apply the results in the context of an individual having a loss of the lower half of their arm and who were in need of a transradial prosthesis.

The way in which I conducted my research, allowed me to look beyond age, and explore the benefits through a clinical perspective. I did this by utilizing three individuals, as well as using a similar testing method as used in [1], because Olsen's research shows that this is a better way to analyze clinical measurements such as usage and benefits.

Before contacting my participant P1, my research method was approved by my school's Institutional Review Board (IRB). Once approved I reached out to P1, and asked if they would be willing to participate in my research. I gave P1 and an informed consent sheet. The informed consent clearly stated exactly what P1 would be doing, how their confidentiality will be kept, and any potential risks that may happen in the research, as well as the right to say no at any time. The participant was required to have a parent signature due to being underage along with their own because the participant is a part of a protected group of minors, and some with disabilities.

Once willing, the participant signed the consent form, I received prior information from P1 about their time with a traditionally built transradial prosthesis, and arranged a day for testing. On the test, I measured the dimensions in centimeters of the patient's arm, this allowed me to digitally create a 3-D printed prosthetic transradial prosthesis. The arm was made on an Ender 3 V2 3-D printer from my home. At the computer station I used Creality 3-D, and put in the design measurement allowing me to design a prosthetic arm on a CAD file. Once completed I recorded how long it took me to do so, following that I compared that to P1's statement of how long it took for them to receive their traditionally built transradial prosthesis. For the testing there were 10 questions and the sheet that was given to P1 who had to write down which transradial prosthesis they preferred, and why they preferred one over the other. The questions went as followed:

1. How comfortable do you find the socket?

2. Is the socket secure? Does it feel like it could slip off your arm?

3. Are there any tough spots on the socket? (Bumps, causing redness, raw skin, and or rubbing too much/too tight)

4. How long can you actively use the device without any discomfort?

5. What are your thoughts on the socket compared to the original device?

These next steps are filled out for P1 of this paper and have been modified to the process used. Digital manufacturing has many different steps that can be switched out, or shortened for the process as well as benefiting the patient's time. The steps of a digital manufacturing process include:

6. Patient History P1: Initial consultation to gather information on previously made traditional upper-limb sockets and identifying why they were no longer being worn, then asking open ended questions on what the patient wanted with having a 3D printed upper-limb device.

7. Limb preparation: Using a measuring sheet from the $3 \mathrm{D}$ printing website, enable printing designs through thingiverse. I marked areas of concern on the paper along with $\mathrm{cm}$ measurements from around the bicep to the end of the residual limb.

8. Limb shape capture: Measured with measuring tape by $\mathrm{cm}$ to determine limb size and proper fitting for prosthetics device. 
9. Initial modification: Combine one forearm piece to a different model of a hand to better suit the patient's needs.

10. Diagnostic socket manufacturing: Used PLA plastic for original arm made, as well as for the second arm, which included more modifications.

11. Further modification: Modified the bending of the wrist and comfort on the triceps piece, changed string from braided fishline to a fabric coated elastic band for better ROM, and moved up the olecranon piece slightly to allow for better ROM.

12. Modified positive model creation: This is performed to improve the pressure distribution, some of the pressure can be redistributed by removing areas on the prosthetic device [6].

13. Final additions: It is important that the prosthetics keep track of follow-up appointments around every 4-6 months for maintenance and modifications [9].

According to [1], steps five, six, and seven are optional in the traditional and digital manufacturing processes, but not skipping them will lead to a better fitting upper-limb socket. Another tip is when step five is thoroughly detailed the prosthetists can combine the steps in eight and nine when the fabrication process arrives.

For comparison, listed below are general steps for a traditionally manufactured transradial prosthesis. It is best to keep in mind that although there are different amounts of steps the timeframe is divergent.

1. Assessing the client's needs and conditions. After this the prosthetists can then decide what type of device will be needed, and which parts they will need when making it.

2. The prosthetist will check existing muscles of the patient's residual limb, and see if they are suited to control a prosthetic device. For the traditional method this can be measured directly on the skin.

3. The socket must be shaped in this step to allow a prosthesis to fit the client's body. This means the prosthetics would take a custom mold of the patient's residual limb, this ensures how the new prosthesis will fit.

4. For this step the patient would come back on a different day, at this visit the socket is checked to make sure it fits the client's body well, and that the arm is able to function well within the socket allowing good ROM (range of motion).

5. The rest of the prosthesis is then fabricated.

6. Modified positive model creation.

7. During this step, the final prosthesis is then fit and small adjustments are made to fine-tune the device and its control as well as patient handling. This can be a very simple task, or depending on how many adjustments are truly needed it can be quite a hectic one.

The traditional build of an upper-limb prosthesis is quite a lengthy process compared to a digital manufacturing process using a 3D printer. Although, it is not necessarily the steps that are more difficult, most research studies emphasize the time frame difference between the two methods. This section went over the process of a traditional upper-limb prosthesis drafted by [5]. 
A

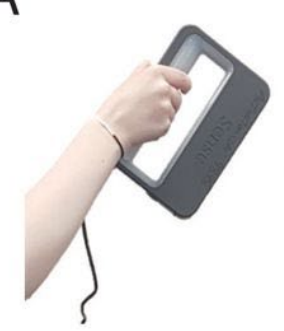

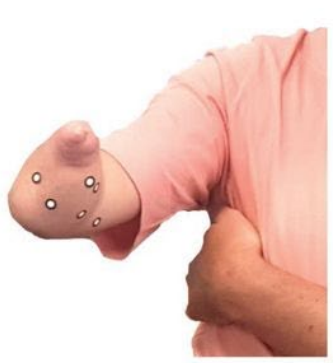

B
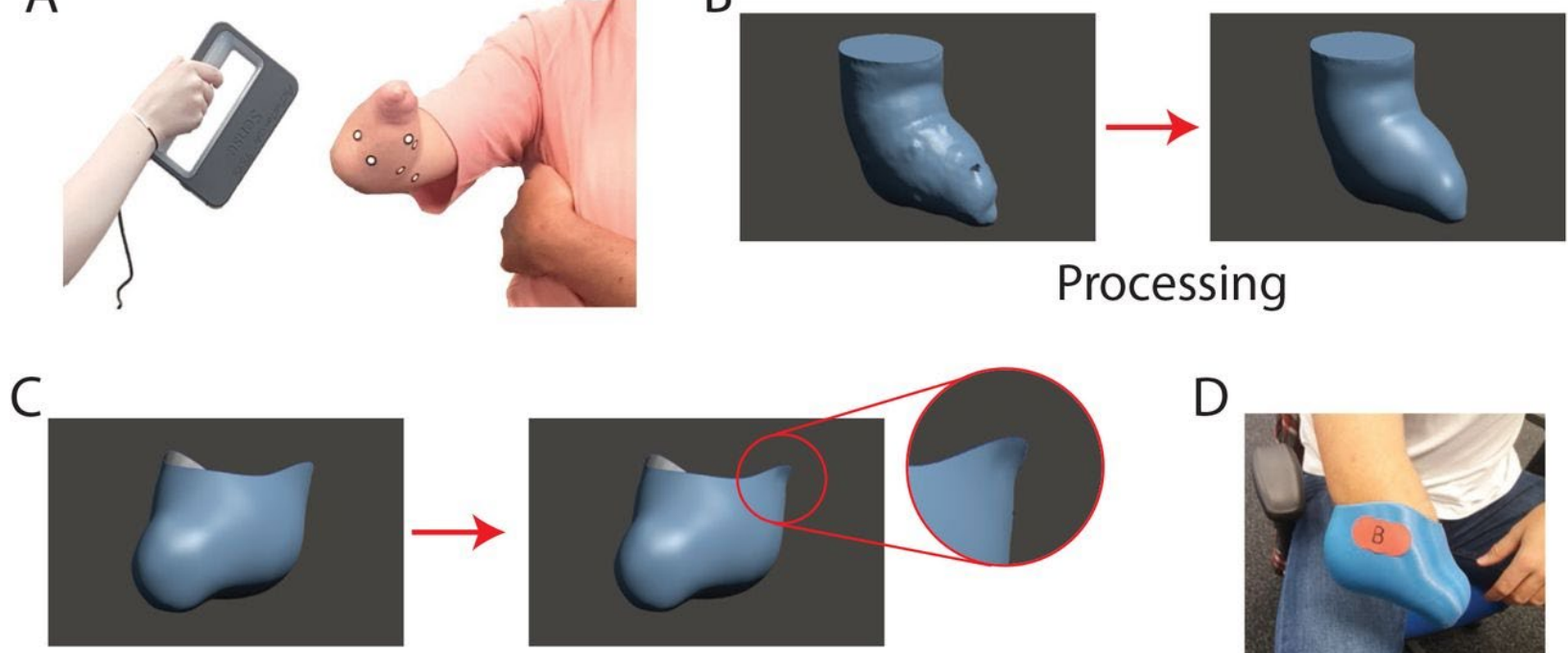

D

\section{Modification}

Figure 3: explaining what the picture means of the process of using a 3D printer

The image presented above is only a reference, for this specific research I used a tape measure, to measure the centimeters of P1's arm, the images presenting the printing process are similar but still a reference. Clearly, it is not the same arm being shown. This project used a full arm to print, and the images only show an elbow device that will eventually add a forearm and hand.

\subsection{Interview}

All participants took part in an interview. The interview included a set of questions but the discussion was kept openended. The questions were:

Set 1: P1 was given the following questions along with three possible answers to choose from, those questions included:

14. How comfortable do you find the socket?

15. Is the socket secure? Does it feel like it could slip off your arm?

16. Are there any tough spots on the socket? (Bumps, causing redness, raw skin, and or rubbing too much/too tight)

17. How long can you actively use the device without any discomfort?

18. What are your thoughts on the socket compared to the original device?

From the twelve-month process of being able to use the original arm, what are any complaints or recommendations you have for your second one? After explaining why, I made the modifications I did, the participant is to answer in the following form: "This is or is not a good idea because... Instead, I would have preferred this because... or this would have benefited me because... or I would have liked this or have liked to see you do this, this or this for the modification process..."

Set 2 was given to P1 and was for the patient to compare the unmodified/slight modification to the 2nd modified arm. This question was given twice to have the two different answers and then for me to compare them in the results section. 
This table included:

\begin{tabular}{|l|l|l|l|}
\hline Comfort & Uncomfortable & Tolerable & Comfortable \\
\hline Slippage/secure fit & Unsecure & Adjustment required & Secure \\
\hline Socket issues & Socket modification issues & Needs a slight adjustment & Fits great \\
\hline Tolerability use & Not wearing & Occasional/as needed & Daily \\
\hline Comparison & Worse & No change & Better/improved \\
\hline
\end{tabular}

\section{Results}

The first report of this section will go over costs and what materials were used and why I chose to use them (section 3.1), and the time investment required by clinician and patient to manufacture the socket with a 3D digital method (3.1.1). As well as the PDFs for the files and the modification files (3.1.2), and I will then provide further feedback from the interview responses from some participants, which will lean more towards P1 (3.2). Finally, the paper will discuss the results of the research.

\subsection{Quantitative analysis}

The modified arm for P1 was replicated on an Ender 3 V2 3D printer. This printer is relatively low at cost and is an upgraded printer from the original Ender 3. The printer used for this project included a tempered glass bed, which is better for prints and maintains the heat beautifully compared to other printers on the market that have fluctuating heat settings throughout the printing time, which can possibly affect the object sticking and can cause a misprint. When using the CAD files off of e-Nable, [6] the files were brought up on Thingiverse and were able to be printed through a MacBook Pro. With the printer, the company provides an SD card and USB that can be inserted in the laptop I was using (and is also capable of being inserted into any device being used except mobile devices), which then allowed me to download the printing filed onto the SD card and insert it into the printer allowing me to print the files. The files used can be found in 3.1.2.

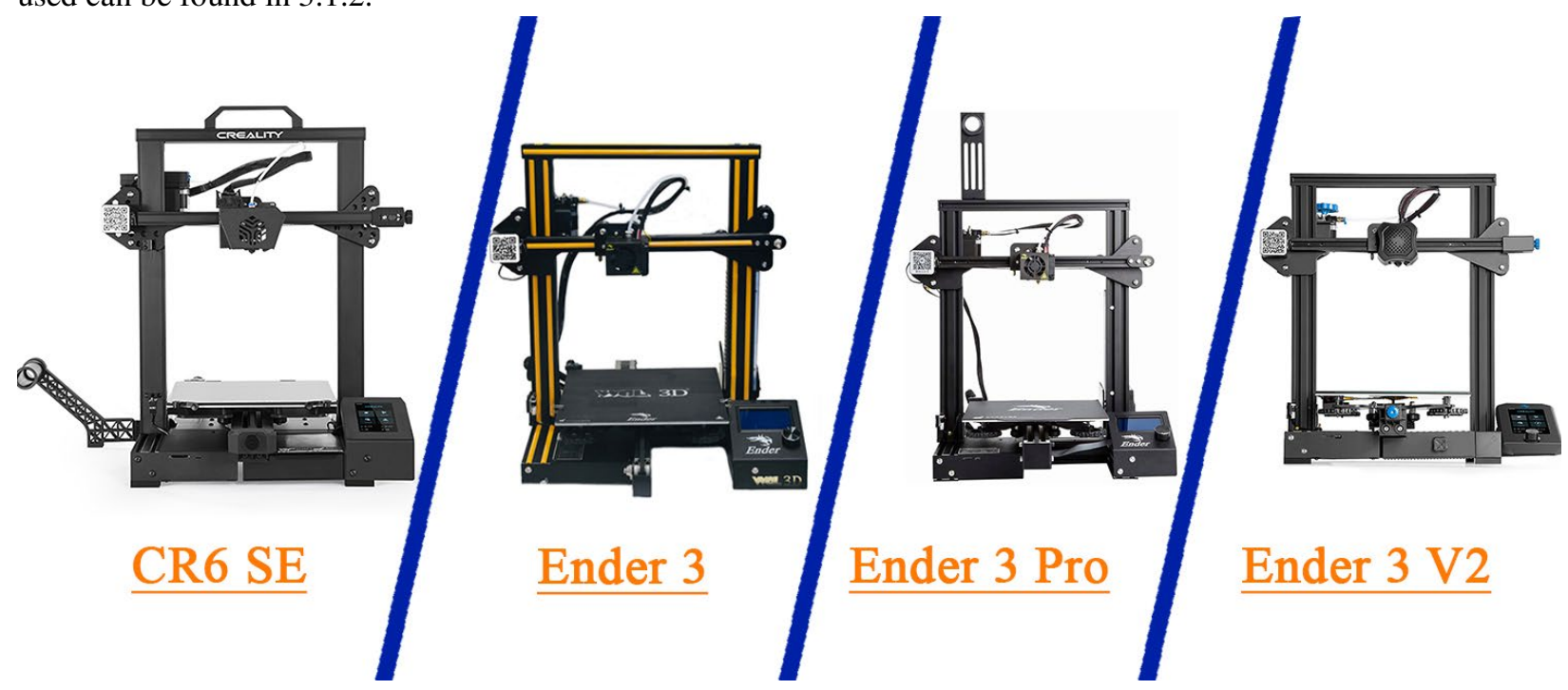

Figure 4: Representation image on previous printers compared to the Ender 3 V2 


\subsubsection{Time investment}

The original arm made took all together approximately 21 hours. The process as a whole with having to work with 3 school schedules took 29 days total and 22 days dismissing weekends. During this time P1 made an additional comment at the end

...The process of 3D printing was much faster than the traditional build of the arm and so far, it works fantastic and functions very smoothly.

Due to not having differing school schedules when making the second arm, it was completed in 5 days.

\subsubsection{PDF files and modifications}

File References:

The Raptor Reloaded - Enabling The Future

Raptor Reloaded by e-NABLE by e-NABLE

File Modifications:

e-NABLE Device Color Customizer

E-Nable Pin Adjustable tendons by bkgray115

https://www.thingiverse.com/make:233742

\subsection{Summary of interview responses}

The responses typed below represent the explanation typed in italic to show direct quotes from the participants. The questions given during the interview process were to achieve ambiguous answers for the results. The results given created categorical data, which were used in the table and charts when identifying how integrating 3D printing can be successful in the field of prosthetics. Results will be summarized in the chart below. Images of the original prosthetic for P1 can be found at the end of the paper, as well as the more modified arm for P1. CAD files from the website Enable will be included in 3.1.2.

\subsubsection{Comfort}

The 3 participants found their unmodified prosthetic device to be comfortable and well-fitted. For P1 the feedback on the modified socket was no change from the unmodified socket, with P2 and P3 also stating that they slightly favored the digital over the traditional although they were both well-fitted and comfortable according to P1: 


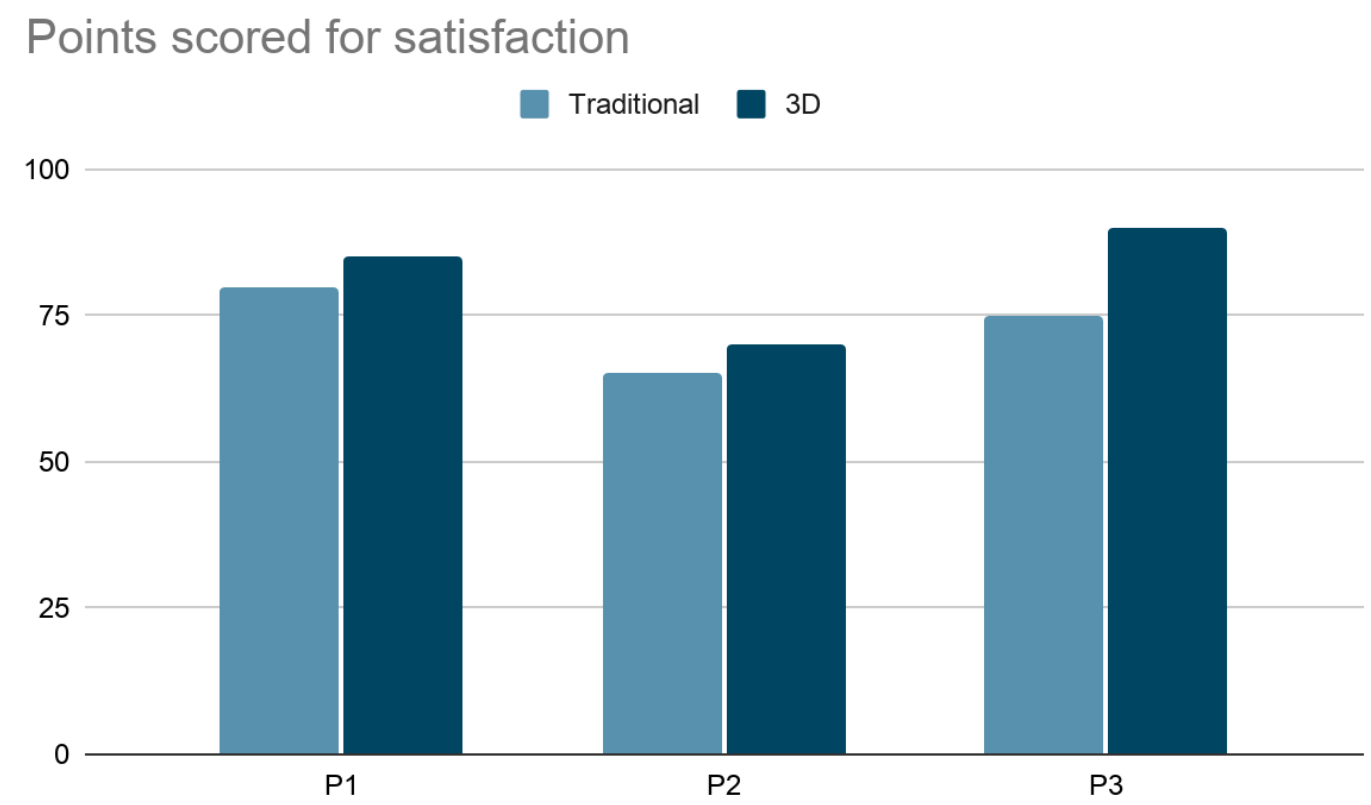

...I can use this arm daily if I want to, it is comfortable.

All-embracing, P1 rated comfort high, and according to [1] her comfort scale also scored high with having her patients highlight minor problems.

\subsubsection{Suspension}

The suspension scores were high on the digital prosthetic device I designed, finding both the traditional and digital to be a secure fit.

...This is (socket) very secure on me, it is great that it is adjustable to how I want it.

Although different measuring devices were used between the two studies P3 stated:

...it's not secure and can be taken off easily [1]

\subsubsection{Socket modification problems}

Although the socket was initially rated comfortable it was identified that minor modifications needed to be made for P1. The chief complaint was the olecranon, and how it can be slightly painful when attempting to meet full ROM.

...minor spot issues, near the elbow (olecranon), can hurt very slightly when maximum range of motion is met.

\subsubsection{Tolerability}

Participants were asked how long they could actively use the prosthetic arm without any discomfort. In my primary data sample, P1 claimed: 
...I can wear it up to the 1-2 hour marking period, it could not be worn and actively used all day.

This issue brought up by P1 has the explanation of the slight discomfort when attempting to achieve full ROM (listed above in 3.2.3).

\subsubsection{Comparison to original socket before modifications}

After carefully listening to the issues identified with P1's socket, I went back to the CAD files and made more modifications. I made the decision to the olecranon by slightly moving it upwards to better fit the participants measurements, allowing for better ROM. Although this was not an issue brought up by the participant, when making the original wrist joint, the braided fishline would rub hard when using the wrist multiple times in a row. In the modification process I chose to change the suspension placement on the wrist. To do this, I changed the CAD file so the fishline did not have to be knotted on the bicep and instead had a maze-like box on the top on the wrist joint allowing for adjustment of the strings to avoid breakage and increase safety. The modification for the wrist was changed without patient complaint. The modification included 53D rectangles coming out of a rounded rectangle on the wrist piece to allow for better ROM (range of motion). Having done this also allowed for the strings to last longer without wearing down from contact. The reference for this description is located in figure 5 The braided fishline was swapped with a fabricated elastic string allowing for better flexion and extension.

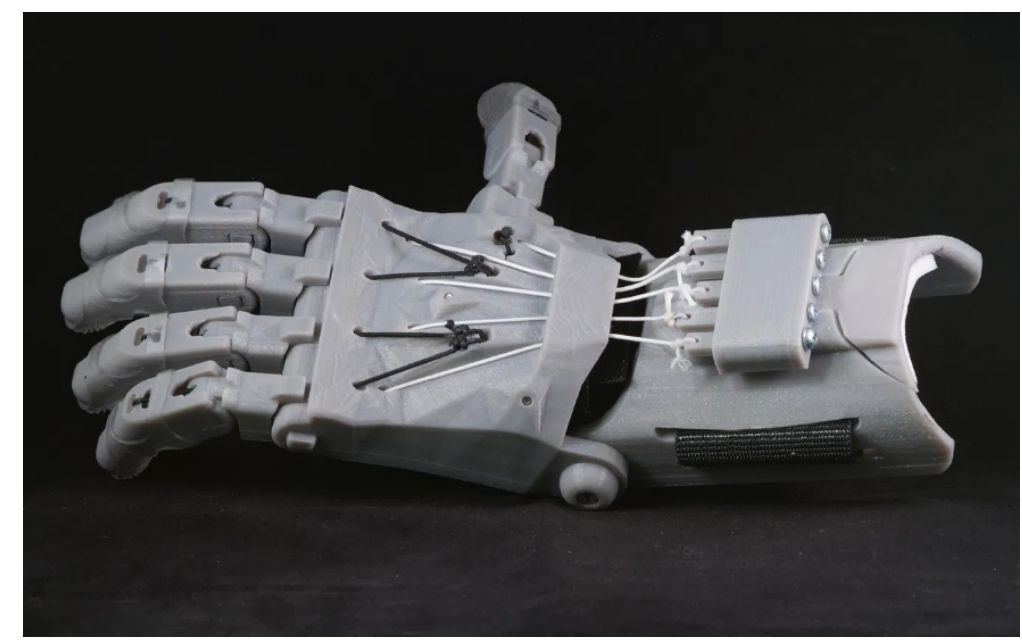

Figure 5: Further clarity on the modification made for the wrist

\subsubsection{Additional comments}

After the interview process, P1 had further commentary that concluded with:

...considering the timeframe, cost, and the fact that those involved in the manufacturing process weren't professionals with 20 years of experience, you guys did extremely well in the making of this arm, and I applaud you all for it.

\subsubsection{Overall result summary}

Overall, the socket was found to be comfortable, it did have concerns and ended up containing minor issues, which were fixed in the second modification process. In P2 and P3 pulled from another study to be compared with P1, they found it to also be comfortable but brought up concerns with the suspension and gave examples on how to improve 
the socket for future use. P1 vocalized the great stability before and after the modifications, and reduced pain after the modification of the olecranon. As the final results came up, it proved how the goal of this research was thoroughly met. The goal was to prove that 3D printing is more beneficial than a traditionally built prosthesis by comparing the two methods through a case study. By looking at the commentary, and graphs it is clearly shown how it was a positive outcome for the gap in my research. All three patients claimed in their comments how although both the traditional prosthetic and the digital prosthetic device fit wonderfully and they could use it for the time given, they still continued to prefer the digital arm over the traditionally manufactured one.

\section{Clinician commentary}

During the session with P1, having a lack of engineering experience in a clinician's point of view contributed to the time frame of having the arm 3D printed from the CAD files, at first the participant needed a few more minor adjustments (adding Velcro, filing rough spots, tightening the braided fishline for the suspension) it overall fit nice and P1 was satisfied for the time being. According to the paper I am comparing to, it was stated by P2 that "the wings, trimlines, and proximal contouring seemed to be the main problem" [1]. Having modifications made to an already fitted prosthetic device can have very different outcomes regarding comfort and stability. Having done a modification, it is more based on the experience the clinician has, and their views on being able to tell what exactly is causing these issues. Most methods of modifications in this medical field are difficult to translate, mostly due to every patient being different. Each patient will come in with their own set of concerns regarding what modifications they would like done, having case specific patients makes it difficult to base another study off of this career field.

\section{Discussion}

This section will specifically discuss the learning aspects of the study of research conducted, and will also include a comparison of J.E. Hanger to modern manufacturing. In conclusion, this paper will explore the implications of this research study.

\subsection{Analysis of experimental results and the interview}

This process has proven the much-shortened time gap between a traditional build and a 3D modeled build. From the results of the interview process some of the participants reported that they preferred the unmodified socket but still proved that 3D printing is a more integrative approach. Identified in section 2.3, although the steps are the same, it is more beneficial to the clinician's time as well as the patient's time. Most individuals want their prosthesis sooner rather than later especially when adapting later in life. The summary of the interview responses reported that the 3D model was a more secure and better fit, although it needed minor modifications for some the time frame still proved the goal of the research.

\subsection{Further work and limitations/implications}

Due to this being a high school in a small town in eastern Michigan, there were not many participants in the area who had had a congenital deformity or amputation at the transradial level. For this reason, P2 and P3 were pulled from a similar study that this paper is based off of [1]. For a future process to be done, limitations and implications could apply to having access to a 3D printing device as well as the software needed for this process to be successful. Located in section 3.1, and represented in figure 4 is the model printer used for this process. Another implication was not having made a traditional manufactured transradial prosthetic arm myself, although P1 stated that they had had multiple traditional arms prior to the 3D printed prosthetic arm, and preferred the 3D, it may have skewed my results by 
not having made the traditional arm myself. Although the 3D arm had minor issues, they were corrected within a 5hour time frame, and the patient still received the arm they day they were supposed to.

\section{Outlook}

This section will be for discussing the many contrasting perspectives on the legality and safety of manufacturing prosthetic devices using a 3D printer, and will also discuss the positives and negatives of integrating 3D printing into the field of O\&P.

\section{1 research perspective}

From a researcher's perspective, this project was appreciable. Being the researcher of the complex topic and career field, it was a good challenge to prove to myself how the integration of 3D printing would be beneficial to the field of orthotics and prosthetics. This research proved to have the outcome I had wanted. That outcome being that the three participants preferred the digital arm over the traditional arm even with their dissimilar ages. Doing research on a future career field is beneficial in shaping the mindset of the doctor a person wishes to become, as well as wanting to change perspective in this persistently changing career field and research topic.

\section{2 clinical perspective}

From a clinical perspective, being the clinician of this case opened up a broader mindset of the various options on this researched topic. Having read numerous scholarly articles, both similar and different to this one, it allowed me to understand why there is an opposing view. Being a clinician to this research also allowed me to go into more depth with what I chose to present. Essentially proving how integrating 3D printing into prosthetics and orthotics would manifest the benefits, and it did.

\section{3 potential benefits}

This project allowed for the research to show the many benefits of integrating digital manufacturing into prosthetics. One of the advantages is the customization of the limb. Whether scanning the residual limb or hand measuring it with a tape measure the 3D process allows for customization of color and many different designs which recently have become more popular over the past couple years. The 3D printers specialize in form-fitting outlines allowing for a better fitted prosthetic, which also decreases slippage. Speaking of the traditional process, the manufacturing process involved doesn't allow for accurate geometry that is required for the customization that could easily be done with 3D manufacturing. According to Raise 3D,

“...designers have full control over the specifications of the final product's geometry. 3D printing can produce a design with intricate geometry, which can include organic shapes, cavity, structures, and lightweight construction".

Predominantly, integrating 3D printing will allow for both patients and clinicians to receive and produce fully custom products in a much shorter time span than a traditional manufacture of a prosthetic limb. To list another benefit, the cost is significantly reduced. A traditional transradial prosthesis costs approximately $\$ 3,000$ to $\$ 30,000$ [9]. For most individuals to look at that proven range they are most likely mind boggled, and even with the most expensive traditionally built arm on the market it will only last 3-5 years. Better yet, there is a solution. As this paper has presented numerous times, using a 3D printer can reduce that cost. Most 3D printers used for prosthetic devices range 
from around $\$ 30$ to $\$ 50$ all the way to $\$ 500$. Of course, it can be more expensive depending on which printer is chosen off the market, it is still a significant decrease. Those huge bills and costs have essentially been diminished [7].

\section{Conclusion}

The clear outlook for implementing the usage of 3D printing manufacturing techniques is vivid. There is rapid interlinkage and many custom design work that will benefit patients. Currently, 3D printing is being used but it still needs to be implemented more. There are more benefits related to 3D printing limbs that outweigh traditional manufacturing by a landslide. Clearly stated in the commentary section are the comments of how the patients benefited from the usage of 3D printing. In the near future as more literature reviews and research is presented for clinical assessment of electromyographic 3D printing of bionic arms, 3D printing will take off even faster than it already has. Having this will mean more accurate geometry and outlines for future prosthetic limbs, as well as adding more choices of limb replacement into the field of prosthetics and orthotics [8].

\section{Acknowledgments}

I would like to thank all who helped me achieve this research project, all contributions were considered greatly. I would like to give thanks to my expert advisor Professor Rebecca Spragg (Eastern Michigan University) for the useful tips and information, as well as my high school AP Research: Capstone teacher Mrs. Kimberly Tetmeyer. I would also like to give a special acknowledgement to my friends and family who supported me all year and pushed me to great accomplishments with this research project, listed as follows; Bryanna Furby and Gavin Bowlin. As well as the endless support from my wonderful parents Douglas Mortier and Susan Layton.

\section{Bibliography}

[1] J. Olsen, S. Day, S. Dupan, K. Nazarpour, and M. Dyson, "3D-Printing and upper-limb prosthetic sockets; promises and pitfalls," 2020.

[2] A. Thompson, "Who was James Hanger and what was the 'Hanger limb'?," Daily mail, Daily Mail, 19-Feb2018.

[3] A. Manero et al., "Implementation of 3D printing technology in the field of prosthetics: Past, present, and future,” Int. J. Environ. Res. Public Health, vol. 16, no. 9, p. 1641, 2019.

[4] “The J.E. Hanger Story,” Hanger.com. [Online]. Available: http://www.hanger.com/history/Pages/The-J.E.Hanger-Story.aspx. [Accessed: 18-Apr-2021].

[5] "Prosthetics Fabrication and Fitting," Limbclinic.com, 06-Dec-2012. [Online]. Available: https://limbclinic.com/prosthetics-fabrication-and-fitting.php. [Accessed: 18-Apr-2021].

[6] "4: Prosthetic Management: Overview, Methods, and Materials: O\&P Virtual Library," 4: Prosthetic

Management: Overview, Methods, and Materials | O\&P Virtual Library. [Online]. Available: http://www.oandplibrary.org/alp/chap04-01.asp. [Accessed: 13-May-2021]. 
[7] P. D. Rhonda Turner, "Prosthetics Costs: The High Price of Prosthetic Limbs," Disabled World, 02-Dec-2020. [Online]. Available: https://www.disabled-world.com/assistivedevices/prostheses/prosthetics-costs.php. [Accessed: 13-May-2021].

[8] Editor, "Why Will 3D Printing Enhance The Prosthetics Market? - Raise3D: Reliable, Industrial Grade 3D Printer," Raise3D. [Online]. Available: https://www.raise3d.com/case/why-will-3d-printing-enhance-theprosthetics-

market/\#: : :text=3D\%20printing $\% 20$ gives $\% 20$ prosthetics $\% 20$ unique, $\mathrm{a} \% 20$ high $\% 20$ degree $\% 20$ of $\% 20$ customization. [Accessed: 13-May-2021].

[9] K. F. Gretsch, H. D. Lather, K. V. Peddada, C. R. Deeken, L. B. Wall, and C. A. Goldfarb, "Development of novel 3D-printed robotic prosthetics for transradial amputees," Prosthetics \& Orthotics International, vol. 40, no. 3, pp. 400-403, 2016. 